\title{
Self-Organizing Flying Drones with Massive MIMO Networking
}

\author{
Zhangyu Guan ${ }^{\dagger}$, Nan Cen ${ }^{\dagger}$, Tommaso Melodia ${ }^{\dagger}$, and Scott Pudlewski ${ }^{\ddagger}$ \\ ${ }^{\dagger}$ Department of Electrical and Computer Engineering, Northeastern University, Boston, MA 02115, USA \\ ${ }^{\ddagger}$ Air Force Research Laboratory (AFRL), Rome, NY 13440, USA \\ Email: \{zguan,ncen, melodia\}@ece.neu.edu,scott.pudlewski.1@us.af.mil
}

\begin{abstract}
This article studies distributed algorithms to control self-organizing swarm drone hotspots with massive MIMO networking capabilities - a network scenario referred to as OrgSwarm. We attempt to answer the following fundamental question: what is the optimal way to provide spectrally-efficient wireless access to a multitude of ground nodes with mobile base stations/aerial relays mounted on a swarm of drones and endowed with a large number of antennas; when we can control the position of many-antenna-enabled drones, access association of ground nodes to drones, and the transmit power of ground nodes?

The article first derives a mathematical formulation of the problem of spectral efficiency maximization through joint control of the movement of many-antenna-enabled aerial drones, access association of single-antenna ground nodes to many-antenna drones, and transmit power of ground nodes. It is shown that the resulting network control problem is a mixed integer nonlinear nonconvex programming problem (MINLP). We then first design a distributed solution algorithm with polynomial computational complexity. Then, a centralized but globally optimal solution algorithm is designed based on a combination of the branch and bound framework and convex relaxation techniques to provide a performance benchmark for the distributed algorithm. Results indicate that the distributed algorithm achieves a network spectral efficiency very close (over $95 \%$ on average) to the global optimum.
\end{abstract}

\section{INTRODUCTION}

The proliferation of innovative Internet of Things (IoT) applications, including intelligent transportation, wireless video surveillance, augmented reality, among others, has resulted in increasing demand for faster wireless communication links with higher spectral efficiency; as well as techniques to reduce interference between co-located links operating on the same spectrum bands and hence to increase the spectral efficiency [1]-[6]. This paper studies OrgSwarm, a new approach to provide ground connectivity by exploring infrastructure-less self-organizing swarm drone hotspots with massive MIMO networking.

With OrgSwarm, a swarm of many-antenna-enabled aerial drones collaboratively provide data collection/forwarding services to a group of single-antenna ground ad hoc nodes, and send to ground nodes control commands that are generated either locally at the drones or in a remote fusion center.

This work is based upon material supported in part by Air Force Research Laboratory under Contract FA8750-14-1-0074 and Grant ARMY W911NF17-1-0034.

ISBN 978-3-903176-05-8 (c) 2018 IFIP
We attempt to provide an answer to the following question: What is the best way to provide spectrally-efficient wireless access/networking to a group of ground nodes with mobile base stations/aerial relays mounted on drones and endowed with a large number of antennas; when you can control the movement of drones, access association, and transmit power of ground nodes? We attempt to answer this compelling question by maximizing the spectral efficiency of OrgSwarm by jointly considering three tightly coupled network control strategies:

- Movement control of drones. Compared to infrastructurebased cellular networks with static massive MIMO base stations as in [7], [8], a peculiar feature of OrgSwarm is that many-antenna-enabled aerial drones need to move dynamically to adapt to changes in the location or traffic demands of ground nodes; nodes leaving or joining the network; as well as time and spatially-varying interference level, among others, to provide coverage with higher spectral efficiency.

- Access association of ground nodes to drones. This is jointly determined by several factors, including network topology (i.e., the relative locations of aerial drones and ground nodes), wireless channel quality currently experienced by ground nodes and transmit power of ground nodes, among others. Moreover, in a massive MIMO setting, a transmission is typically conducted in two phases: pilot-based channel estimation and data transmission. The maximum number of ground nodes associated to an aerial drone is constrained by the length (in symbols) of the pilot sequences used in the channel estimation phase [9], [10].

- Massive-MIMO power control. In OrgSwarm, all ground nodes are allowed to operate over the entire available spectrum band and at any transmission time. Therefore, it is imperative to regulate the transmit power of ground nodes to eliminate mutual interference in both channel estimation and data transmission phases caused by imperfect channel orthogonalization in the case of limited number antennas at each aerial drone, and hence to maximize the achievable SINR and spectral efficiency.

Clearly, the above three network control problems are tightly coupled and should therefore be jointly considered to obtain the optimal network operating point. Because of the binary ground-drone association variables (which will be clearer 
in Section III), the resulting OrgSwarm control problem is a mixed integer nonlinear nonconvex programming problem (MINLP), which is generally NP-hard and there is no existing solution algorithm that can be used to obtain the globally optimal solution with polynomial computational complexity. This paper makes the following main contributions:

- OrgSwarm control formulation. We formulate mathematically the OrgSwarm control problem with control objective of maximizing the spectral efficiency of OrgSwarm by jointly controlling the movement of aerial drones, ground-drone association and transmit power of ground nodes.

- Distributed solution algorithms. Distributed solution algorithms are designed to solve the formulated OrgSwarm control problem based on primal decomposition, which decomposes the resulting MINLP problem into three distributed sub-problems: single-antenna-to-many-antennas access association, massive MIMO power control, and drone movement.

- Globally optimal solution algorithm. To provide a performance benchmark for the distributed solution algorithm, we design a centralized but globally optimal solution algorithm based on a combination of the branch and bound framework and of convex relaxation techniques that result in an $\varepsilon$-optimal solution of the original MINLP problem, where $\varepsilon$ is a predefined level of optimality precision that can be set arbitrarily close to 1 .

- Performance evaluation. The performance of the proposed distributed solution algorithm is evaluated in terms of network spectral efficiency by comparing it to the global optimum through extensive simulation experiments.

The reminder of the paper is organized as follows. We review related work in Section II, and describe the system model and problem formulation in Section III. In Sections IV and $\mathrm{V}$, we present the distributed solution algorithm and the globally optimal solution algorithm, respectively. Performance evaluation is presented in Section VI, and finally we draw conclusions in Section VII.

\section{RELATED WORK}

There is a large and growing body of literature on unmanned aerial vehicular networking, focusing on UAV-assisted guidance [11], UAV-based data collection [12]-[14] and relaying [15]-[19], ground-aerial channel measurements [20] as well as tracking and control of UAV networks [21]-[23]. Readers are referred to [24]-[27] and references therein for an extensive survey of this research area. Most of these works focus on single-antenna aerial vehicles and conventional MIMO, with very few recent efforts considering massive MIMO [23]. Different from [23], where Chandhar et al. derived the achievable uplink capacity from a many-antennas ground base stations to a set of single-antenna aerial drones, in this paper we maximize the aggregate throughput of single-antenna ground nodes to a set of aerial drones each endowed with a large number of antennas.
Compared to conventional multiuser MIMO, massive MIMO can attain much higher spectral efficiency by using a large number of antennas with low-complexity linear precoding technologies [9], [28]-[33]. For example, in [29], the authors derived an exact achievable rate expression in closedform for maximum-ratio combining/maximum-ratio transmission (MRC/MRT) processing and an analytical approximation of the achievable rate for zero-forcing (ZF) processing for multi-pair full-duplex massive MIMO relay system. In [30], Jin et al. derived ergodic rates for the case with a finite number of antennas and concluded that the ergodic sum-rate can be maintained while the relay power is scaled down by a factor of the number of the antennas at the relay over the number of users. Please refer to [7], [10], [34] for a good survey and tutorial on massive MIMO networking. These papers are focused on infrastructure-based cellular networks with static many-antenna-enabled base stations, and focus on asymptotic performance analysis with respect to a single network parameter (e.g., power). Our paper, instead, considers aerial drone hotspots with massive MIMO capabilities in an infrastructure-less network scenario.

\section{System Model and Problem Statement}

We consider infrastructure-less wireless ad hoc networks with many-antenna-enabled drones, where a set $\mathcal{A}$ of manyantenna-enabled aerial drones are used to collect field information from a set $\mathcal{G}$ of single-antenna ground nodes. The goal of the aerial drones is to collect field information, make action decisions either locally at each drone or by sending the information fusion results to a remote control center, and finally send the action commands back to ground nodes. We focus on information uploading over ground-drone links since it causes only low-level traffic load to transmit information fusion results and control commands back to ground nodes. Our objective is to maximize the network-wide spectral effciency by jointly controlling the movement of aerial drones, associating single-antenna ground nodes to many-antenna drones, and controlling the transmit power of ground nodes.

Ground-Drone Association. We consider single-home accommodation for ground nodes in favor of tractable complexity in modeling and theoretical analysis, i.e., each ground node is associated with at most one drone. Denote the association vector as $\boldsymbol{\alpha} \triangleq\left(\boldsymbol{\alpha}_{g}\right)_{g \in \mathcal{G}}$, where $\boldsymbol{\alpha}_{g} \triangleq\left(\alpha_{g a}\right)_{a \in \mathcal{A}}$ is the association vector of ground node $g \in \mathcal{G}$, with $\alpha_{g a}=1$ if ground node $g$ is associated with drone $a$ and $\alpha_{g a}=0$ otherwise. Denote $\mathcal{A}_{g} \subset \mathcal{A}$ as the set of aerial drones ground node $g$ is associated with, $\mathcal{G}_{a} \subset \mathcal{G}$ as the set of ground nodes associated with drone $a$, and $A_{g}$ and $G_{a}$ as the number of aerial drones in $\mathcal{A}_{g}$ and the number of ground nodes in $\mathcal{G}_{a}$, respectively. Then, we have

$$
\begin{aligned}
& \mathcal{A}_{g} \triangleq\left\{a \mid a \in \mathcal{A}, \alpha_{g a}=1\right\}, \forall g \in \mathcal{G}, \\
& A_{g} \triangleq\left|\mathcal{A}_{g}\right| \leq 1, \forall g \in \mathcal{G}, \\
& \mathcal{G}_{a} \triangleq\left\{g \mid g \in \mathcal{G}, \alpha_{g a}=1\right\}, \forall a \in \mathcal{A}, \\
& G_{a} \triangleq\left|\mathcal{G}_{a}\right| \leq G_{\max }, \forall a \in \mathcal{A},
\end{aligned}
$$


where $G_{\max }$ is the maximum number of ground nodes that can be served by each aerial drone $a \in \mathcal{A} .^{1}$

Ground-Drone Link Capacity. In massive MIMO networks, a transmission is typically accomplished in two phases, i.e., channel estimation and data transmission [9], [10], [36]. In the first phase, ground nodes send a set of orthogonal pilot sequences to their service aerial drones for channel estimation. Denote $\tau$ as the length (in symbols) of each pilot sequence. For ground node $g \in \mathcal{G}$, let $\mathcal{I}_{g}$ represent the set of ground nodes using the same pilot sequences as node $g$, and $a(g)$ denote the service aerial drone of ground node $g$. Let $\mathbf{p}=\left(p_{g}\right)_{g \in \mathcal{G}}$ represent the transmit power vector of ground nodes, with $p_{g}$ being the power of $g$ th ground node in $\mathcal{G}$. Then, the achievable signal-to-interference-plus-noise ratio (SINR) of ground node $g \in \mathcal{G}$, denoted as $\gamma_{g}$, can be written as [37]

$$
\gamma_{g}=\frac{\left(M-G_{a(g)}\right) \tau \rho \beta_{g g}^{2} p_{g} /\left(1+\tau \rho \xi_{g g}\right)}{1+\sum_{g^{\prime} \in \mathcal{G}} \mu_{g^{\prime} g} p_{g^{\prime}}+\frac{\tau \rho\left(M-G_{a(g)}\right) \sum_{g^{\prime} \in \mathcal{I}_{g} \backslash g} \beta_{g^{\prime} g}^{2} p_{g^{\prime}}}{1+\tau \rho \xi_{g g}}}
$$

where $M$ represents the number of antennas available at each aerial drone, $\beta_{g^{\prime} g}$ is the slow fading channel gain between ground node $g^{\prime} \in \mathcal{G}$ to the service aerial drone of ground node $g$, i.e., aerial drone $a(g), \rho$ is transmit signal-to-noise ratio $(\mathrm{SNR}), \xi_{g^{\prime} g} \triangleq \sum_{l \in \mathcal{I}_{g^{\prime}}} \beta_{l g}$, and $\mu_{g^{\prime} g}$ is defined as

$$
\mu_{g^{\prime} g}= \begin{cases}\beta_{g^{\prime} g}, & \text { if } a\left(g^{\prime}\right) \notin \mathcal{A}\left(\mathcal{I}_{g}\right) \\ \beta_{g^{\prime} g}\left(1-\frac{\tau \rho \beta_{g^{\prime} g}}{1+\tau \rho \xi_{g^{\prime} g}}\right), & \text { otherwise, }\end{cases}
$$

with $\mathcal{A}\left(\mathcal{I}_{g}\right) \triangleq\left\{a\left(g^{\prime}\right) \mid g^{\prime} \in \mathcal{I}_{g}\right\}$ representing the set of service aerial drones of interfering ground nodes in $\mathcal{I}_{g}$.

Let $\mathbf{x}=\left(x_{a}\right)_{a \in \mathcal{A}}, \mathbf{y}=\left(y_{a}\right)_{a \in \mathcal{A}}, \widetilde{\mathbf{x}}=\left(\widetilde{x}_{g}\right)_{g \in \mathcal{G}}$ and $\widetilde{\mathbf{y}}=$ $\left(\widetilde{y}_{g}\right)_{g \in \mathcal{G}}$ respectively represent the coordinate vectors of aerial drones and ground nodes, with $x_{a}, y_{a}$ being $\mathrm{x}$ - and $\mathrm{y}$-axis coordinates of aerial drone $a$, and $\widetilde{x}_{g}$ and $\widetilde{y}_{g}$ being $\mathrm{x}-$ and $\mathrm{y}$-axis coordinates of ground node $g$, respectively. Then, the slow fading channel gain $\beta_{g^{\prime} g}$ in (6) can be expressed as

$$
\beta_{g^{\prime} g}=H_{g^{\prime} g}(\mathbf{x}, \mathbf{y}, \widetilde{\mathbf{x}}, \widetilde{\mathbf{y}}) \zeta_{g^{\prime} g},
$$

where $\zeta_{g^{\prime} g}$ represents the log-normal slow fading between ground node $g^{\prime}$ and the service aerial drone of ground node $g$, i.e., aerial drone $a(g), H_{g^{\prime} g} \triangleq d_{g^{\prime} g}^{-\chi}(\mathbf{x}, \mathbf{y})$ is locationdependent path loss with $\chi$ being path loss factor and $d_{g^{\prime} g}^{-\chi}$ being the distance (in meter) between ground node $g^{\prime}$ and aerial drone $a(g)$. Then, the capacity achievable by ground node $g \in \mathcal{G}$, denoted as $R_{g}(\boldsymbol{\alpha}, \mathbf{p}, \mathbf{x}, \mathbf{y}, \widetilde{\mathbf{x}}, \widetilde{\mathbf{y}})$, can be expressed as

$$
R_{g}(\boldsymbol{\alpha}, \mathbf{p}, \mathbf{x}, \mathbf{y}, \widetilde{\mathbf{x}}, \widetilde{\mathbf{y}})=B \log _{2}\left(1+\gamma_{g}\right),
$$

where $B$ is bandwidth (in $\mathrm{Hz}$ ) and $\gamma_{g}=\gamma_{g}(\boldsymbol{\alpha}, \mathbf{p}, \mathbf{x}, \mathbf{y}, \widetilde{\mathbf{x}}, \widetilde{\mathbf{y}})$ is the achievable SINR defined in (5).

\footnotetext{
${ }^{1}$ The maximum number of served ground nodes cannot exceed the number of antennas available to each aerial drone and the length of pilot sequences used in channel estimation [35].
}

Problem Statement. The network control objective can then be stated as maximizing the sum capacity of all ground nodes in $\mathcal{G}$ and hence the network spectral efficiency by jointly determining transmit power vector $\mathbf{p}$ for ground nodes, coordinate vectors $\mathbf{x}$ and $\mathbf{y}$ for aerial drones, and grounddrone association vector $\boldsymbol{\alpha}$. The problem is a mixed integer nonlinear nonconvex programming problem (MINLP) because of the nonlinear nonconcave mathematical expression of $\gamma_{g}$ with respect to $\boldsymbol{\alpha}, \mathbf{p}, \mathbf{x}, \mathbf{y}$ in (5)-(7) and binary association variables $\boldsymbol{\alpha}$. Given an arbitrary such problem, how to design distributed solution algorithms to achieve the global optimum is still an open problem. In the following sections, we first present a distributed solution algorithm in Section IV and then design a centralized but globally optimal solution algorithm in Section $\mathrm{V}$ to provide a performance benchmark for the distributed solution algorithm.

\section{Distributed Solution Algorithm}

A key step of the distributed solution algorithm design is to decompose the original network control problem into a series of subproblems, by solving which in a distributed manner the original problem can be solved. In our case, network control variables $\boldsymbol{\alpha}, \mathbf{p}, \mathbf{x}, \mathbf{y}$ are tightly coupled with each other in the SINR $\gamma_{g}$ expression in (5) and, therefore, in the achievable capacity in (8). This results in the fact that the resulting network control problem is architecturally undecomposable. ${ }^{2}$ Therefore, we design our distributed solution algorithm by decomposing the network control problem following a primal decomposition approach. The core idea is to divide the feasible set of the original problem into multiple parts by fixing a subset of variables at each time; and solve the resulting subproblems iteratively [39]. In our case, primal decomposition can be accomplished by fixing the aerial drone location variables $\mathbf{x}, \mathbf{y}$, association variables $\boldsymbol{\alpha}$, and transmit power vector $\mathbf{p}$, resulting in three subproblems: ground-drone association, ground node power control and drone movement. In the following, we design an iterative distributed solution algorithm, where at each iteration the three subproblems are solved sequentially.

Massive MIMO Association. Let $\mathbf{x}^{(\nu)}$ and $\mathbf{y}^{(\nu)}$ represent the coordinates of aerial drones and $\mathbf{p}^{(\nu)}$ be the transmit power of ground nodes in current iteration $\nu$. Then in iteration $\nu+1$ the objective of the ground-drone association subproblem is to maximize the aggregate capacity of all ground nodes in $\mathcal{A}$ by determining the association vector $\boldsymbol{\alpha}^{(\nu+1)}$ subject to association constraint (2), which is a nonconvex combinatorial problem. Next, inspired by the behaviors of interacting agents in real-time auctions [40], [41], we present an effective Distributed Auction-based Association Strategy (DAAS) by viewing the drones as sellers while the ground nodes as buyers.

The core idea of DAAS is to let ground nodes iteratively compete for association by reporting their own association

\footnotetext{
${ }^{2} \mathrm{~A}$ problem is architecturally decomposable if its dual problem obtained by introducing Lagrange multipliers can be rewritten into a set of subproblems, each of which can be solved locally in a single protocol layer and network device [38].
} 
preferences to nearby aerial drones, which then make association decisions based on the received preference information. Denote $\mathcal{N}_{g}^{(\nu+1)} \subset \mathcal{A}$ as the set of nearby aerial drones of ground node $g \in \mathcal{G}$ (i.e., aerial drones in the communication range of the ground node). Then, the association preference of ground node $g$ with respect to aerial drone $a \in \mathcal{N}_{g}^{(\nu+1)}$, denoted as $\lambda_{g a}$, can be computed as

$$
\lambda_{g a}=\frac{\log \left(1+\gamma_{g a}\right)}{\sum_{a^{\prime} \in \mathcal{N}_{g}^{(\nu+1)}} \log \left(1+\gamma_{g a^{\prime}}\right)},
$$

where $\gamma_{g a^{\prime}}$ represents the interference-free single-input-singleoutput (SISO) SINR, i.e., the SINR achievable with single antenna and without interference from other ground nodes [35], achievable by ground node $g$ if associated to aerial drone $a^{\prime}$. This can be defined as $\left.\gamma_{g a^{\prime}} \triangleq \rho \beta_{g g}\right|_{a(g)=a^{\prime}}$ with $\rho$ being the transmit SNR and $\left.\beta_{g g}\right|_{a(g)=a^{\prime}}$ being the fading coefficient. Denote $\boldsymbol{\lambda}_{a}=\left(\lambda_{g a}\right)_{g \in \widetilde{\mathcal{N}}_{a}}$ as the preference vector aerial drone $a$ receives from its nearby ground nodes in $\widetilde{\mathcal{N}}_{a} \triangleq\left\{g \mid g \in \mathcal{G}, a \in \mathcal{N}_{g}\right\}$. Then, aerial drone $a$ sorts $\boldsymbol{\lambda}_{a}$ in descending order and sends association offers to a preferred set of maximum $G_{\max }$ of ground nodes, as follows,

$$
\boldsymbol{\lambda}_{a}^{\mathrm{dsc}}=(\underbrace{\lambda_{g_{1} a}, \lambda_{g_{2} a}, \cdots, \lambda_{g_{G_{\max }} a}}_{\text {Preferred Ground Nodes }}, \cdots) .
$$

Then, the association offer corresponding to the highest SISO SINR is accepted by a ground node if it receives multiple offers. The above procedures are repeated until no ground node receives multiple association offers.

In (9), the rationale of computing association preference based on interference-free SISO SINR is as follows. In massive MIMO settings, particularly when the number of antennas $M$ is large, the received SINR is dominated by path loss and large scale shadowing and tends to the interference-free SISO SINR as $M \rightarrow \infty$. Therefore, the capacity with interferencefree SISO SINR can serve as a good indication of association preference and can be computed with low computational complexity.

Massive MIMO Power Control. Given the coordinates of aerial drones $\mathbf{x}^{(\nu)}, \mathbf{y}^{(\nu)}$ and association vectors $\boldsymbol{\alpha}^{(\nu+1)}$, the ground node power control subproblem can be expressed as

$$
\begin{array}{cl}
\text { Given : } & \mathbf{x}^{(\nu)}, \mathbf{y}^{(\nu)}, \boldsymbol{\alpha}^{(\nu+1)} \\
\begin{array}{c}
\text { Maximize } \\
\text { Subject to }:
\end{array} & \sum_{g \in \mathcal{G}} R_{g}(\mathbf{p}) \\
& 0 \leq p_{g} \leq p_{0}, \forall g \in \mathcal{G}
\end{array}
$$

where $R_{g}(\mathbf{p})$ is defined in (5)-(8) and $p_{0}$ is the maximum transmit power of ground nodes. As stated in the following theorem, this problem can be approximated as a convex programming problem in the setting of massive MIMO networking, and hence the optimal power control can be achieved with poly-nominal computational complexity.

Proposition 1. In a massive MIMO setting, for given coordinates of aerial drones and association strategies, ground node transmit power control problem in (11) can be approximated as a convex programming problem.
Proof. According to convexity preserving properties, the logarithm of a concave function is a concave function [42]. Therefore, to prove the theorem we only need to show that the achievable capacity $R_{g}$ in (8) is a concave function. In a massive MIMO setting, it is reasonable to assume that the achievable SINR $\gamma_{g}$ in (5) satisfies $\gamma_{g} \gg 1$ since the negative effects of interference and small scale fading can be largely smoothed out [35]. Then, it can be shown that the expression of capacity $R_{g}$ in (8) has the form of minus-log-sum-exp with respect to logarithm transformation of transmit power variables $p_{g}$, which is a concave function [42].

Drone Movement. In this subproblem, each aerial drone determines its own best coordinates to adapt to the changes in association strategies and transmit power of ground nodes caused by solving the previous two subproblems. With newly obtained association vector $\boldsymbol{\alpha}^{(\nu+1)}$ and transmit power vector $\mathbf{p}^{(\nu+1)}$, the subproblem of aerial drone movement can be written, for each aerial drone $a \in \mathcal{A}$, as

$$
\begin{array}{cl}
\text { Given: } & \boldsymbol{\alpha}_{\mathbf{x}, \mathbf{y}}^{(\nu+1)}, \mathbf{p}^{(\nu+1)} \\
\text { Maximize: } & \sum_{g \in \mathcal{G}_{a}} R_{g}(\mathbf{x}, \mathbf{y}), \\
\text { Subject to }: & x_{\min } \leq x_{a} \leq x_{\max }, \\
& y_{\min } \leq y_{a} \leq y_{\max },
\end{array}
$$

where $x_{\min }, y_{\min }, x_{\max }$ and $y_{\max }$ represent the minimum and maximum $\mathrm{x}$ - and $\mathrm{y}$ - coordinates, respectively, and $\mathcal{G}_{a}$ represents the set of ground nodes associated with aerial drone $a$ with given association vector $\boldsymbol{\alpha}^{(\nu+1)}$. In this subproblem, the mathematical expression of utility function $R_{g}(\mathbf{x}, \mathbf{y})$ in (12) has a log-convex form, which is in general a nonconcave function with respect to coordinate variables $\mathbf{x}$ and $\mathbf{y}$. In this paper we solve subproblem (12) using an interior point method [42] to search for locally optimal coordinates for each aerial drone in favor of a low-complexity distributed solution. In Section $\mathrm{V}$, we will design a centralized but globally optimal solution algorithm at the cost of extra computational complexity.

Computational Complexity Analysis. In the distributed solution algorithm, the above three subproblems are solved iteratively and sequentially at each iteration. In the ground-drone association subproblem, the association strategy is determined iteratively. In each iteration, the association variable $\alpha_{g a}$ can be determined for at least one ground node, and therefore the maximum number of associations is $|\mathcal{G}|$, and the overall computational complexity of the association is $\mathcal{O}(|\mathcal{G}|)$. Both the power control and the aerial drone movement subproblems can be solved in polynomial computational complexity, i.e., $\mathcal{O}(|\mathcal{G}||\mathcal{A}|)$. Therefore, the complexity of the overall distributed solution algorithm is $\mathcal{O}(|\mathcal{G}|(|\mathcal{A}|+1))$ for each iteration. The convergence of the proposed distributed solution algorithm will be studied in Section VI based on simulations, where results indicate that the algorithm can converge very fast, i.e., within tens of iterations.

So far, we have presented a distributed solution algorithm to jointly control the movement of aerial drones, ground-drone association and power control of ground nodes 
in infrastructure-less wireless ad hoc networks with manyantenna-enabled aerial drones. A natural question is: How does the distributed solution algorithm compare to the global optimum in terms of aggregate spectral efficiency? In the remaining of the paper we answer this question by designing a centralized but globally optimal solution algorithm to provide a performance benchmark for the distributed solution algorithm.

\section{Globally Optimal Solution Algorithm}

As stated in Section III, the "social" objective of the network control problem is to maximize the network spectral efficiency by jointly controlling movement of aerial drones, the transmit power of ground nodes and the ground-drone association strategies. The social OrgSwarm control problem can be mathematically represented as

$$
\begin{aligned}
& \text { Given: } \mathcal{A}, \mathcal{G}, G_{\max } \\
& \underset{\boldsymbol{\alpha}, \mathbf{p}, \mathbf{x}, \mathbf{y}}{\operatorname{aximize}}: \quad U \triangleq \sum_{g \in \mathcal{G}} R_{g}(\boldsymbol{\alpha}, \mathbf{p}, \mathbf{x}, \mathbf{y}) \\
& \text { Subject to : } 0 \leq p_{g} \leq p_{0}, \forall g \in \mathcal{G} \text {, } \\
& x_{\text {min }} \leq x_{a} \leq x_{\max }, \forall a \in \mathcal{A} \text {, } \\
& y_{\text {min }} \leq y_{a} \leq y_{\max }, \forall a \in \mathcal{A} \text {, } \\
& \text { (1), (2), (3), (4). }
\end{aligned}
$$

In (13), the individual throughput $R_{g}$ is a nonconvex/nonconcave function with respect to coordinates variables $\mathbf{x}, \mathbf{y}$ and transmit power vector $\mathbf{p}$. Moreover, the association variables $\boldsymbol{\alpha}$ take only binary values. Therefore the resulting network control problem is a mixed integer nonlinear nonconvex programming problem (MINLP), for which there is in general no existing solution algorithm that can be used to obtain the global optimum in polynomial computational complexity. In this paper, we design a globally optimal solution algorithm based on a combination of the branch and bound framework and of convex relaxation techniques [43], [44].

\section{A. Overall Algorithm}

Denote $\boldsymbol{\Gamma}_{0}=\{\boldsymbol{\alpha}, \mathbf{p}, \mathbf{x}, \mathbf{y} \mid$ constraints in (13) $\}$ as the feasible set of initial problem (13) and let $U^{*}\left(\boldsymbol{\Gamma}_{0}\right)$ represent the global optimum of problem (13) over $\Gamma_{0}$, then the objective of our algorithm is to iteratively search for a $U$ so that

$$
U\left(\boldsymbol{\Gamma}_{0}\right) \geq \varepsilon U^{*}\left(\boldsymbol{\Gamma}_{0}\right),
$$

where $\varepsilon \in(0,1]$ is predefined optimality precision. To this end, the algorithm maintains a set $\boldsymbol{\Gamma}=\left\{\boldsymbol{\Gamma}_{i}, i=0,1,2, \cdots\right\}$ of subproblems by iteratively partitioning feasible set $\boldsymbol{\Gamma}_{0}$ into a series of smaller subsets (see Section V-C). ${ }^{3}$ The algorithm also maintains a global upper bound $\bar{U}_{\mathrm{glb}}\left(\boldsymbol{\Gamma}_{0}\right)$ and a global lower bound $\underline{U}_{\mathrm{glb}}\left(\boldsymbol{\Gamma}_{0}\right)$ on $U^{*}\left(\boldsymbol{\Gamma}_{0}\right)$ so that

$$
\underline{U}_{\mathrm{glb}}\left(\boldsymbol{\Gamma}_{0}\right) \leq U^{*}\left(\boldsymbol{\Gamma}_{0}\right) \leq \bar{U}_{\mathrm{glb}}\left(\boldsymbol{\Gamma}_{0}\right)
$$

to drive the iterations of subproblem partitions, as follows.

- Global upper bound $\bar{U}_{\mathrm{glb}}\left(\boldsymbol{\Gamma}_{0}\right)$ : For each subproblem $\boldsymbol{\Gamma}_{i} \in \boldsymbol{\Gamma}$, the algorithm computes a local upper bound

\footnotetext{
${ }^{3}$ In this paper we use $\boldsymbol{\Gamma}_{i}$ to refer to both subproblem $i$ and the corresponding feasible set.
}

$\bar{U}_{\mathrm{lcl}}\left(\boldsymbol{\Gamma}_{i}\right)$ on network utility function $U$ via convex relaxation (see Section V-B). Then the global upper bound $\bar{U}_{\text {glb }}\left(\boldsymbol{\Gamma}_{0}\right)$ can be updated as

$$
\bar{U}_{\mathrm{glb}}\left(\boldsymbol{\Gamma}_{0}\right)=\max _{\boldsymbol{\Gamma}_{i} \in \boldsymbol{\Gamma}}\left\{\bar{U}_{\mathrm{lcl}}\left(\boldsymbol{\Gamma}_{i}\right)\right\}
$$

- Global lower bound $\underline{U}_{\mathrm{glb}}\left(\boldsymbol{\Gamma}_{0}\right)$ : Similarly, for each subproblem $\boldsymbol{\Gamma}_{i} \in \boldsymbol{\Gamma}$ a local lower bound $\underline{U}_{\mathrm{lcl}}\left(\boldsymbol{\Gamma}_{i}\right)$ is computed based on the solution obtained by solving the relaxed convex network control problem. Then the global lower bound $\underline{U}_{\mathrm{glb}}\left(\boldsymbol{\Gamma}_{0}\right)$ can be updated as

$$
\underline{U}_{\mathrm{glb}}\left(\boldsymbol{\Gamma}_{0}\right)=\max _{\boldsymbol{\Gamma}_{i} \in \boldsymbol{\Gamma}}\left\{\underline{U}_{\mathrm{lcl}}\left(\boldsymbol{\Gamma}_{i}\right)\right\}
$$

The algorithm terminates if $\underline{U}_{\mathrm{glb}}\left(\boldsymbol{\Gamma}_{0}\right) \geq \varepsilon \bar{U}_{\mathrm{glb}}\left(\boldsymbol{\Gamma}_{0}\right)$ is reached and the global optimum $U^{*}\left(\boldsymbol{\Gamma}_{0}\right)$ is set to $U^{*}\left(\boldsymbol{\Gamma}_{0}\right)=\bar{U}_{\mathrm{glb}}\left(\boldsymbol{\Gamma}_{0}\right)$ as a upper-bound benchmark. Otherwise, the algorithm selects a subproblem from $\Gamma$ and further partitions its feasible set into two smaller subsets, computes local upper and lower bounds and updates the global bounds $\bar{U}_{\mathrm{glb}}\left(\boldsymbol{\Gamma}_{0}\right)$ and $\underline{U}_{\mathrm{glb}}\left(\boldsymbol{\Gamma}_{0}\right)$ as in (16) and (17), respectively. In our algorithm, we select the subproblem $\Gamma_{i} \in \Gamma$ with the highest local upper bound to partition, i.e.,

$$
\boldsymbol{\Gamma}_{i}=\underset{\boldsymbol{\Gamma}_{i}}{\arg \max } \bar{U}_{\mathrm{lcl}}\left(\boldsymbol{\Gamma}_{i}\right)
$$

Based on the global bounds update criterion in (16) and (17), the gap between the two global bounds converges to 0 as the partition progresses. Furthermore, from (15), $\underline{U}_{\mathrm{glb}}\left(\boldsymbol{\Gamma}_{0}\right)$ and $\bar{U}_{\mathrm{glb}}\left(\boldsymbol{\Gamma}_{0}\right)$ converge to the global optimum $U^{*}\left(\boldsymbol{\Gamma}_{0}\right)$.

\section{B. Convex Relaxation}

For each subproblem $\boldsymbol{\Gamma}_{i} \subset \boldsymbol{\Gamma}$, which is MINLP in our case, a key step is to obtain a relaxed but convex version of $\boldsymbol{\Gamma}_{i}$ so that it is easy to compute a tight local upper bound $\bar{U}_{\mathrm{lcl}}\left(\boldsymbol{\Gamma}_{i}\right)$. In this paper the convex relaxation is designed following a two-phase approach as follows.

Phase 1: In this phase the relaxation is accomplished by assuming i) there is no mutual interference among ground nodes, i.e., interference items in the denominator of (5) are set to zero, and that all ground nodes use different pilot sequences in channel estimation and hence $\xi_{g g}=0$ in (5); ii) the maximum number of ground nodes that can be associated to an aerial drone is not limited to $G_{\max }$ in (4). Then, the objective of the relaxed network control problem is to maximize the aggregate capacity of ground nodes by determining the optimal coordinate $\mathbf{x}$ and $\mathbf{y}$ of drones, i.e.,

$$
\begin{array}{cl}
\underset{\mathbf{x}, \mathbf{y}}{\operatorname{Maximize}:} & U \triangleq \sum_{g \in \mathcal{G}} R_{g}(\mathbf{x}, \mathbf{y}) \\
\text { Subject to }: & x_{\min } \leq x_{a} \leq x_{\max }, \forall a \in \mathcal{A}, \\
& y_{\min } \leq y_{a} \leq y_{\max }, \forall a \in \mathcal{A},
\end{array}
$$

where $R_{g}(\mathbf{x}, \mathbf{y})=B \log _{2}\left(1+\gamma_{g}(\mathbf{x}, \mathbf{y})\right)$ with $\gamma_{g}(\mathbf{x}, \mathbf{y})$ defined 
in (5). Since $\gamma_{g}(\mathbf{x}, \mathbf{y}) \gg 1, R_{g}(\mathbf{x}, \mathbf{y})$ can be approximated as

$$
\begin{aligned}
R_{g}(\mathbf{x}, \mathbf{y}) & \approx B \log _{2}\left(\gamma_{g}(\mathbf{x}, \mathbf{y})\right) \\
& \leq B \log _{2}\left(M \tau \rho p_{0} \zeta_{g g}^{2} H_{g g}^{2}(\mathbf{x}, \mathbf{y})\right) \\
& =B \log _{2}\left(\frac{M \tau \rho p_{0} \zeta_{g g}^{2}}{d_{g g}^{\chi}(\mathbf{x}, \mathbf{y})}\right) \\
& =B \log _{2}\left(M \tau \rho p_{0} \zeta_{g g}^{2}\right)-\chi B \log _{2}\left(d_{g g}(\mathbf{x}, \mathbf{y})\right),
\end{aligned}
$$

where the inequality in (21) holds since $G_{a(g)} \geq 0$ in (5), $\chi$ is path loss factor and $d_{g g}(\mathbf{x}, \mathbf{y})$ is distance (in meter) from ground node $g$ to its service aerial drone $a(g)$.

Since $d_{g g}(\mathbf{x}, \mathbf{y})$ in (23) is a convex Euclidean norm with respect to $\mathbf{x}$ and $\mathbf{y}[42], \log _{2}\left(d_{g g}(\mathbf{x}, \mathbf{y})\right.$ cannot be theoretically guaranteed to be concave. In this phase, we obtain a convex relaxation of (23) based on linear approximation of logarithmic function. To this end, we first replace $d_{g g}(\mathbf{x}, \mathbf{y})$ in (23) with $t$, then $\log _{2}\left(d_{g g}(\mathbf{x}, \mathbf{y})\right.$ in (23) can be represented as $\log _{2}(t)$ subject to $t \geq d_{g g}(\mathbf{x}, \mathbf{y})$. Then, $\log _{2}(t)$ can be further relaxed using a set of linear functions using a segment and three tangent lines.

Phase 2: Phase 2 of relaxation is invoked if the algorithm is done with partitioning coordinate variables $\mathbf{x}$ and $\mathbf{y}$, i.e., for each aerial drone $a \in \mathcal{A}$,

$$
\begin{aligned}
& x_{\max , a}-x_{\min , a} \leq \Delta x, \\
& y_{\max , a}-y_{\min , a} \leq \Delta y,
\end{aligned}
$$

where $x_{\max , a}$ and $x_{\min , a}\left(y_{\max , a}\right.$ and $\left.y_{\min , a}\right)$ are upper and lower bounds of $\mathrm{x}$-axis coordinate $x_{a}$ (y-axis coordinate $y_{a}$ ), and $\Delta x$ and $\Delta y$ are predefined movement step size of aerial drones in $\mathrm{x}$ - and $\mathrm{y}$-axis, respectively. The objective in this phase is to determine the optimal association vector $\boldsymbol{\alpha}$ with given aerial drones coordinates vectors $\mathbf{x}^{*}$ and $\mathbf{y}^{*}$ and without considering mutual interference among ground nodes as in Phase 1 relaxation. Let $R_{g a}$ represent the capacity achievable by ground node $g \in \mathcal{G}$ if $g$ is associated to aerial drone $a \in \mathcal{A}$, then the optimal association can be obtained by solving the

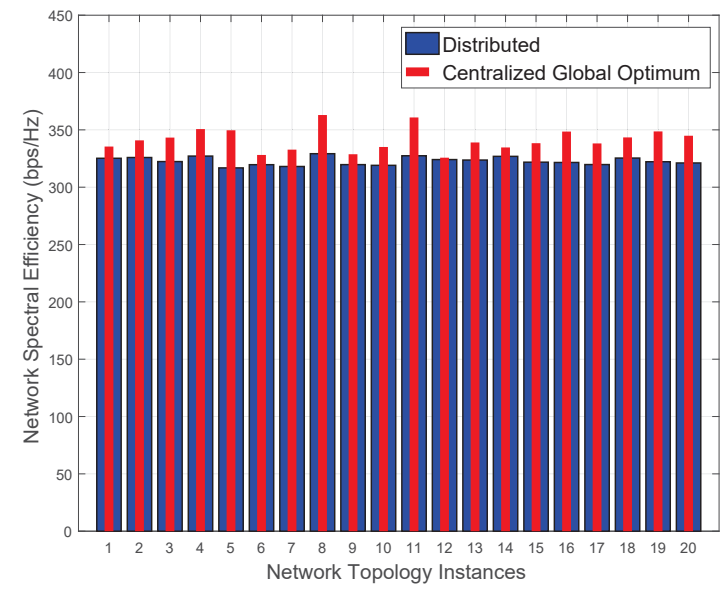

Fig. 1: Aggregate network spectral efficiency achievable by distributed solution algorithm and global optimum. following linear optimization problem:

$$
\begin{array}{cl}
\begin{array}{c}
\text { Given: } \\
\text { Maximize : }
\end{array} & \sum_{\boldsymbol{\alpha} \in \mathcal{A}} \sum_{g \in \mathcal{G}} \mathbf{y}_{g a} R_{g a}\left(\boldsymbol{\alpha}, \mathbf{x}^{*}, \mathbf{y}^{*}\right) \\
\text { Subject to : } & 0 \leq \alpha_{g a} \leq 1, \forall a \in \mathcal{A}, g \in \mathcal{G}, \\
& \sum_{g \in \mathcal{G}} \alpha_{g a} \leq G_{\max }, \forall a \in \mathcal{A}, \\
& \sum_{a \in \mathcal{A}} \alpha_{g a} \leq 1, \forall g \in \mathcal{G} .
\end{array}
$$

As variable partition progresses, the association variable $\alpha_{g a}$ becomes fixed either to 0 or 1 in all subproblems, for which the optimal transmit power $\mathbf{p}$ can be obtained by solving a convex optimization problem as in Section IV.

\section{Variable Partition}

Variable partition can be conducted by partitioning association variable $\boldsymbol{\alpha}$ and movement variables $\mathbf{x}$ and $\mathbf{y}$. For example, given a subproblem $\boldsymbol{\Gamma}_{i} \in \boldsymbol{\Gamma}$, by fixing association variable $\alpha_{g a}$ subproblem $\boldsymbol{\Gamma}_{i}$ can be partitioned into two subproblems with feasible sets $\boldsymbol{\Gamma}_{i, 1}=\left\{(\boldsymbol{\alpha}, \mathbf{p}, \mathbf{x}, \mathbf{y}) \in \boldsymbol{\Gamma}_{i} \mid \alpha_{g a}=0\right\}$ and $\boldsymbol{\Gamma}_{i, 2}=\left\{(\boldsymbol{\alpha}, \mathbf{p}, \mathbf{x}, \mathbf{y}) \in \boldsymbol{\Gamma}_{i} \mid \alpha_{g a}=1\right\}$, respectively. For movement variables, say $x_{a} \in\left[\begin{array}{ll}x_{\min , a} & x_{\max , a}\end{array}\right]$ for aerial drone $a \in \mathcal{A}$, the partition can be conducted by splitting $x_{a}$ from the half, resulting in two subproblems with feasible sets

$$
\begin{aligned}
& \boldsymbol{\Gamma}_{i, 1}=\left\{(\boldsymbol{\alpha}, \mathbf{p}, \mathbf{x}, \mathbf{y}) \in \boldsymbol{\Gamma}_{i} \mid x_{a} \in\left[\begin{array}{ll}
x_{\min , a} & x_{\operatorname{mid}, a}
\end{array}\right]\right\}, \\
& \boldsymbol{\Gamma}_{i, 2}=\left\{(\boldsymbol{\alpha}, \mathbf{p}, \mathbf{x}, \mathbf{y}) \in \boldsymbol{\Gamma}_{i} \mid x_{a} \in\left[\begin{array}{ll}
x_{\operatorname{mid}, a} & x_{\max , a}
\end{array}\right]\right\},
\end{aligned}
$$

where $x_{\operatorname{mid}, a} \triangleq \frac{x_{\min , a}+x_{\max , a}}{2}$.

\section{Vi. Performance Evaluation}

We evaluate the performance of the proposed solution algorithms by considering a network area of $1000 \times 1000 \mathrm{~m}^{2}$, with $N_{g}=\{4, \cdots, 15\}$ ground nodes served by $N_{a}=\{2,3\}$ aerial drones. The altitude of the drones are set to 100 meters. The number of antennas of each aerial drone is set to $N_{\text {ant }}=\{20,40, \cdots, 160\}$, the maximum transmit power of each ground node $p_{0}$ is set to $\{100,200, \cdots, 600\} \mathrm{mW}$, path loss factor is set to $\chi=2$, and the average noise power

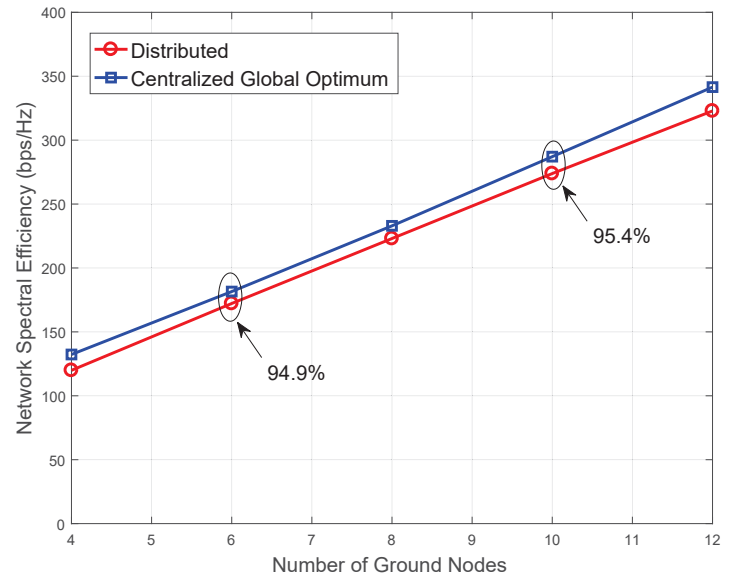

Fig. 2: Network spectral efficiency in the case of different number of ground nodes. 


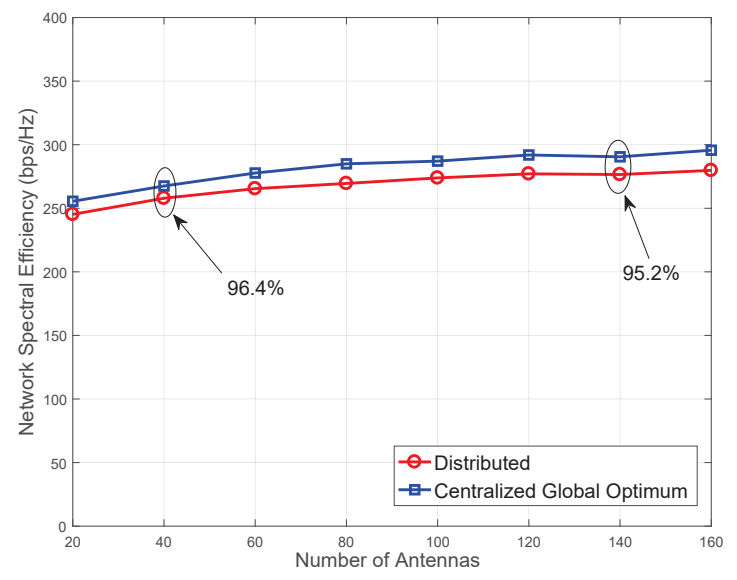

Fig. 3: Network spectral efficiency in the case of different number of aerial drone antennas.

is set to $10^{-8} \mathrm{~mW}$. Results are obtained by averaging over 20 independent simulation instances with network topology randomly generated.

Performance of the proposed distributed solution algorithm is evaluated by comparing it with the global optimum obtained by the centralized solution algorithm designed in Section V. Figure 1 reports the network spectral efficiency achievable by the distributed solution algorithm and the centralized global optimum. It can be seen that the distributed solution algorithm achieves a performance very close to the global optimum in all the tested 20 instances, where 12 ground nodes and 2 aerial drones each with $M=100$ antennas are considered. The average performance of the achievable network spectral efficiency is reported in Fig. 2 with different number of ground nodes. Results indicate that around 95\% percent of the global optimum can be achieved by distributed solution algorithm, e.g., $94.9 \%$ and $95.4 \%$ with 6 and 10 ground nodes, respectively.

The network spectral efficiency is reported in Fig. 3 with the number of antennas at each aerial drone varying from 20 to 160 at step of 20 . It can be seen that over $95 \%$ of the global optimum can be achieved by the distributed solution algorithm. Results also indicate that the achievable network spectral efficiency monotonically increases with the number of antennas but at a decreasing speed. With more than 100 antennas, the effect of increasing the number antennas is marginal.

In Fig. 4 we plotted the achievable network spectral efficiency against the maximum transmit power of ground nodes. Over $95 \%$ of the global optimum can be achieved by the distributed solution algorithms. We noticed that the network spectral efficiency rises only around $10 \%$ by increasing the maximum transmit power by 6 times from 100 to $600 \mathrm{~mW}$. This is because in massive MIMO setting the network basically operates at high SINR regime, i.e., in bandwidth-limited regime. We further studied the effects of different network control strategies on the achievable network spectral efficiency in Fig. 5, where the joint network control strategy is compared

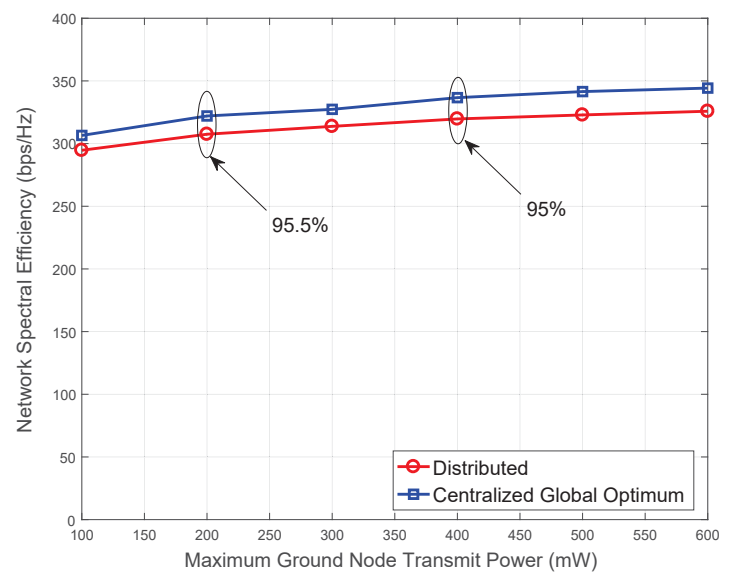

Fig. 4: Aggregate network spectral efficiency against the maximum transmit power of ground nodes.

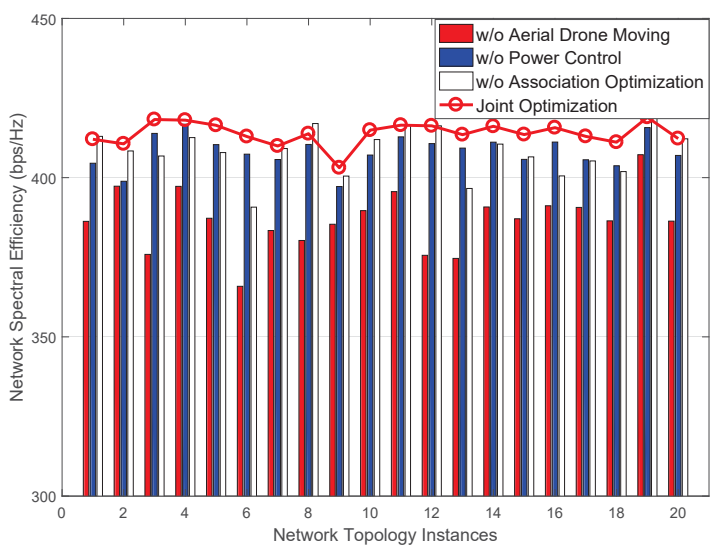

Fig. 5: Achievable network spectral efficiency with different network control strategies.

to the other three strategies, in which aerial drone movement is randomly generated in "w/o Aerial Drone movement", power control and association are executed only once in "w/o" power control and "w/o" association, respectively. It can be seen that the joint network control achieves the highest spectral efficiency in almost all tested instances. Results also indicate that a spectral efficiency close to that of joint control can be achieved by "w/o power control" in most network instances since the network operates in high SINR regime in massive MIMO setting.

\section{CONCLUSIONS}

We studied infrastructure-less wireless ad hoc networking with many-antenna-enabled aerial drone hotspots. The network control objective is to maximize network-wide spectral efficiency by jointly controlling movement of drones, associating single-antenna ground nodes to many-antenna drones, and adapting transmit power of ground nodes. The network control problem was formulated as an integer nonlinear nonconvex programming problem (MINLP). Both distributed and globally optimal solution algorithms have been designed and evaluated with extensive simulation results. Results indicated that the distributed solution algorithm can achieve around $95 \%$ of the global optimum. 


\section{REFERENCES}

[1] M. Noura and R. Nordin, "A Survey on Interference Management for Device-to-Device (D2D) Communication and Its Challenges in 5G Networks," Journal of Network and Computer Applications (Elsevier), vol. 71, pp. 130-150, August 2016.

[2] S. Ali and A. Ahmad, "Resource Allocation, Interference Management, and Mode Selection in Device-to-Device Communication: A Survey," Emerging Telecommunications Technologies, pp. 1-36, March 2017.

[3] Z. Guan and T. Melodia, "CU-LTE: Spectrally-Efficient and Fair Coexistence Between LTE and Wi-Fi in Unlicensed Bands," in Proc. of IEEE INFOCOM, San Francisco, CA, USA, April 2016.

[4] Z. Guan, T. Melodia, and G. Scutari, "Distributed Queuing Games in Interference-limited Wireless Networks," in Proc. of IEEE International Conference on Communications (ICC), Budapest, Hungary, June 2013.

[5] F. Chen, W. Su, S. N. Batalama, and J. D. Matyjas, "Joint Power Optimization for Multi-source Multi-destination Relay Networks," IEEE Transactions on Signal Processing, vol. 59, no. 6, pp. 2370-2381, May 2011.

[6] F. Chen, W. Su, D. A. Pados, J. D. Matyjas, and M. J. Medley, "Relay Location Optimization for Differential Amplify-and-Forward Cooperative Relaying," in Proceedings of IEEE Global Telecommunications Conference, December 2015.

[7] E. Björnson, E. G. Larsson, and T. L. Marzetta, "Massive MIMO: Ten Myths and One Critical Question," IEEE Communications Magazine, vol. 54, no. 2, pp. 114-123, February 2016.

[8] Y. Wu, R. Schober, D. W. K. Ng, C. Xiao, and G. Caire, "Secure Massive MIMO Transmission With an Active Eavesdropper," IEEE Trans. on Info. Theory, vol. 62, no. 7, pp. 3880-3900, July 2016.

[9] E. G. Larsson, O. Edfors, F. Tufvesson, and T. L. Marzetta, "Massive MIMO For Next Generation Wireless Systems," IEEE Communications Magazine, vol. 52, no. 2, pp. 186-195, February 2014.

[10] T. L. Marzetta, "Massive MIMO: An Introduction," Bell Labs Technical Journal, vol. 20, pp. 12-22, 2015.

[11] V. Sharma and R. Kumar, "A Cooperative Network Framework for Multi-UAV Guided Ground Ad Hoc Networks," Journal of Intelligent \& Robotic Systems, vol. 77, pp. 629-652, 2015.

[12] I. Jawhar, NaderMohamed, J. Al-Jaroodi, and S. Zhang, "A Framework for Using Unmanned Aerial Vehicles for Data Collection in Linear Wireless Sensor Networks," Journal of Intelligent \& Robotic Systems, vol. 74, pp. 437-453, 2014.

[13] S. Kandeepan, K. Gomez, L. Reynaud, and T. Rasheed, "AerialTerrestrial Communications: Terrestrial Cooperation and EnergyEfficient Transmissions to Aerial Base Stations," IEEE Trans. on Aerospace and Electronic Systems, vol. 50, no. 4, pp. 2715-2735, Oct. 2014.

[14] K. Daniel, S. Rohde, N. Goddemeier, and C. Wietfeld, "Cognitive Agent Mobility for Aerial Sensor Networks,' IEEE Sensors Journal, vol. 11, no. 11, pp. 2671-2682, Nov. 2011.

[15] F. Ono, H. Ochiai, and R. Miura, "A Wireless Relay Network Based on Unmanned Aircraft System With Rate Optimization," IEEE Trans. on Wireless Communications, vol. 15, no. 11, pp. 7699-7708, Nov. 2016.

[16] L. Sboui, H. Ghazzai, Z. Rezki, and M.-S. Alouini, "Achievable Rates of UAV-Relayed Cooperative Cognitive Radio MIMO Systems," IEEE Access, accepted for publication.

[17] P. Ladosz, H. Oh, and W.-H. Chen, "Optimal Positioning of Communication Relay Unmanned Aerial Vehicles in Urban Environments," in Proc. of International Conference on Unmanned Aircraft Systems (ICUAS), Arlington, VA USA, June 2016.

[18] D. Orfanus, E. P. de Freitas, and F. Eliassen, "Self-Organization as a Supporting Paradigm for Military UAV Relay Networks," IEEE Communications Letters, vol. 20, no. 4, pp. 804-807, April 2016.

[19] L. Ferranti, F. Cuomo, S. Colonnese, and T. Melodia, "Drone Cellular Networks: Enhancing the Quality of Experience of Video Streaming Applications," Ad Hoc Networks (Elsevier), in press, 2018.

[20] T. J. Willink, C. C. Squires, G. K. Colman, and M. T. Muccio, "Measurement and Characterization of Low-Altitude Air-to-Ground MIMO Channels," IEEE Trans. on Vehicular Technology, vol. 64, no. 4, pp. 2637-2648, April 2016.

[21] S. Morgenthaler, T. Braun, Z. Zhao, T. Staub, and M. Anwander, "UAVNet: A Mobile Wireless Mesh Network Using Unmanned Aerial Vehicles," in Proc. of International Workshop on Wireless Networking \& Control for Unmanned Autonomous Vehicles: Architectures, Protocols and Applications, Anaheim, California, Dec. 2012.
[22] Y. Zhou, M. Chen, and C. Jiang, "Robust Tracking Control of Uncertain MIMO Nonlinear Systems with Application to UAVs," IEEE/CAA Journal of Automatica Sinca, vol. 2, no. 1, pp. 25-32, Jan. 2015.

[23] P. Chandhar, D. Danev, and E. G. Larsson, "Massive MIMO as Enabler for Communications with Drone Swarms," in Proc. of International Conference on Unmanned Aircraft Systems (ICUAS), Arlington, VA USA, June 2016

[24] L. Gupta, R. Jain, and G. Vaszkun, "Survey of Important Issues in UAV Communication Networks," IEEE Communications Surveys \& Tutorials, vol. 18, no. 2, pp. 1123-1152, Second Quarter 2016.

[25] E. Y. Samira Hayat and R. Muzaffar, "Survey on Unmanned Aerial Vehicle Networks for Civil Applications: A Communications Viewpoint," IEEE Communications Surveys \& Tutorials, vol. 18, no. 4, pp. 26242661, Fourth Quarter 2016.

[26] M. Erdelj, E. Natalizio, K. R. Chowdhury, and I. F. Akyildiz, "Help from the Sky: Leveraging UAVs for Disaster Management," IEEE Pervasive Computing, vol. 16, no. 1, pp. 24-32, Jan.-March 2017.

[27] Y. Zeng, R. Zhang, and T. J. Lim, "Wireless Communications with Unmanned Aerial Vehicles: Opportunities and Challenges," IEEE Communications Magazine, vol. 54, no. 5, pp. 36-42, May 2016.

[28] T. L. Marzetta, "Noncooperative Cellular Wireless with Unlimited Numbers of Base Station Antennas," IEEE Transactions on Wireless Communications, vol. 9, no. 11, pp. 3590-3600, November 2010.

[29] H. Q. Ngo, H. A. Suraweera, M. Matthaiou, and E. G. Larsson, "Multipair Full-Duplex Relaying With Massive Arrays and Linear Processing," IEEE JSAC, vol. 32, no. 9, pp. 1721-1737, September 2014.

[30] S. Jin, X. Liang, K. K. Wong, X. Gao, and Q. Zhu, "Ergodic Rate Analysis for Multipair Massive MIMO Two-Way Relay Networks," IEEE Transactions on Wireless Communications, vol. 14, no. 3, pp. 1480-1491, March 2015.

[31] G. Amarasuriya, "Sum Rate Analysis for Multi-User Massive MIMO Relay Networks," in Proc. IEEE Global Communications Conference (GLOBECOM), December 2015, San Diego, CA, USA.

[32] J. Chen, X. Chen, W. H. Gerstacker, and D. W. K. Ng, "Resource Allocation for a Massive MIMO Relay Aided Secure Communication," IEEE Transactions on Information Forensics and Security, vol. 11, no. 8, pp. 1700-1711, August 2016.

[33] Z. Zhang, Z. Chen, M. Shen, and B. Xia, "Spectral and Energy Efficiency of Multipair Two-Way Full-Duplex Relay Systems With Massive MIMO," IEEE JSAC, vol. 34, no. 4, pp. 848-863, April 2016.

[34] D. C. Araújo, T. Maksymyuk, A. L. de Almeida, T. Maciel, J. ao C.M. Mota, and M. Jo, "Massive MIMO: Survey and Future Research Topics," IET Communications, vol. 10, no. 15, pp. 1938-1946, October 2016.

[35] H. Q. Ngo, E. G. Larsson, and T. L. Marzetta, "Energy and Spectral Efficiency of Very Large Multiuser MIMO Systems," IEEE Trans. on Commun., vol. 61, no. 4, pp. 1436-1449, April 2013.

[36] L. Lu, G. Y. Li, A. L. Swindlehurst, A. Ashikhmin, and R. Zhang, "An Overview of Massive MIMO: Benefits and Challenges," IEEE Journal of Selected Topics in Signal Processing, vol. 8, no. 5, pp. 742-758, October 2014.

[37] H. Yang and T. L. Marzetta, "Capacity Performance of Multicell Largescale Antenna Systems," in Proc. Allerton Conference on Communication, Control, and Computing, Monticello, IL, Oct. 2013.

[38] M. Chiang, S. H. Low, A. R. Calderbank, and J. C. Doyle, "Layering as Optimization Decomposition: A Mathematical Theory of Network Architectures," Proceedings of the IEEE, vol. 95, no. 1, pp. 255-312, January 2007.

[39] D. P. Palomar and M. Chiang, "A Tutorial on Decomposition Methods for Network Utility Maximization," IEEE Journal on Selected Areas in Communications, vol. 24, no. 8, pp. 1439-1451, August 2006.

[40] R. McAfee and J. McMillan, "Auctions and Bidding," J. Economic Literature, vol. 25, no. 2, pp. 699-738, June 1987.

[41] B. Gerkey and M. Mataric, "Sold!: Auction Methods for Multirobot Coordination," IEEE Trans. Robotics and Automation, vol. 18, no. 5, pp. 758-768, Oct. 2002.

[42] S. Boyd and L. Vandenberghe, Convex Optimization. Cambridge University Press, 2004.

[43] E. L. Lawler and D. E. Wood, "Branch-And-Bound Methods: A Survey," Operations Research, vol. 14, no. 4, pp. 699-719, Jul.-Aug. 1966.

[44] H. D. Sherali and W. P. Adams, A Reformulation-Linearization Technique for Solving Discrete and Continuous Nonconvex Problems. Boston: MA: Kluwer Academic, 1999. 